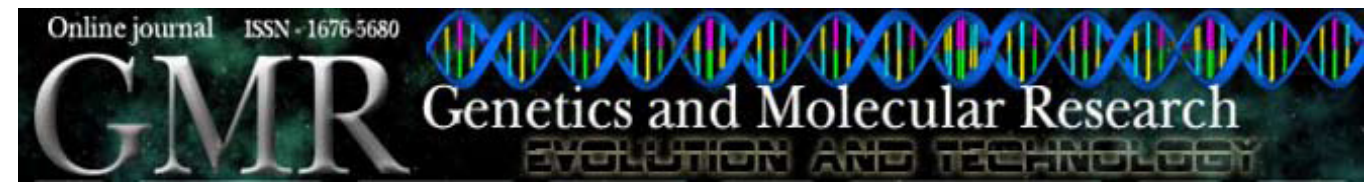

\title{
Identification of three distinguishable phenotypes in golden retriever muscular dystrophy
}

\author{
C.E. Ambrósio ${ }^{4}$, L. Fadel ${ }^{1}$, T.P. Gaiad ${ }^{1}$, D.S. Martins ${ }^{1}$, K.P.C. Araújo ${ }^{1}$, \\ E. Zucconi ${ }^{2}$, M.P. Brolio ${ }^{1}$, R.F. Giglio ${ }^{5}$, A.C. Morini ${ }^{1}$, T. Jazedje ${ }^{2}$, \\ T.R. Froes ${ }^{3}$, M.L.T. Feitosa ${ }^{1}$, M.C. Valadares ${ }^{2}$, P.C.B. Beltrão-Braga ${ }^{1}$, \\ F.V. Meirelles ${ }^{4}$ and M.A. Miglino \\ ${ }^{1}$ Departamento de Cirurgia, Faculdade de Medicina Veterinária e Zootecnia, \\ Universidade de São Paulo, São Paulo, SP, Brasil \\ ${ }^{2}$ Departamento de Genética e Biologia Evolutiva, Instituto de Biociências, \\ Universidade de São Paulo, Centro de Estudos do Genoma Humano, \\ São Paulo, SP, Brasil \\ ${ }^{3}$ Faculdade de Medicina Veterinária, Universidade Federal do Paraná, \\ Curitiba, PR, Brasil \\ ${ }^{4}$ Departamento de Ciências Básicas, \\ Faculdade de Zootecnia e Engenharia de Alimentos, \\ Universidade de São Paulo, São Paulo, SP, Brasil \\ ${ }^{5}$ University of Florida, Gainesville, FL, USA \\ Corresponding author: C.E. Ambrósio \\ E-mail: ceambrosio@usp.br
}

Genet. Mol. Res. 8 (2): 389-396 (2009)

Received January 6, 2009

Accepted February 20, 2009

Published April 7, 2009

ABSTRACT. Duchenne muscular dystrophy (DMD) is a human disease characterized by progressive and irreversible skeletal muscle degeneration caused by mutations in genes coding for important muscle proteins. Unfortunately, there is no efficient treatment for this disease; it causes progressive loss of motor and muscular ability until death. The canine model (golden retriever muscular dystrophy) is similar to DMD, showing similar clinical signs. Fifteen dogs were followed from birth and closely observed for clinical signs. Dogs had their disease status confirmed by polymerase chain reaction analysis and genotyping. Clinical observations of musculoskeletal, morphological, gastrointestinal, respiratory, cardiovascular, and 
renal features allowed us to identify three distinguishable phenotypes in dystrophic dogs: mild (grade I), moderate (grade II) and severe (grade III). These three groups showed no difference in dystrophic alterations of muscle morphology and creatine kinase levels. This information will be useful for therapeutic trials, because DMD also shows significant, inter- and intra-familiar clinical variability. Additionally, being aware of phenotypic differences in this animal model is essential for correct interpretation and understanding of results obtained in pre-clinical trials.

Key words: Duchenne muscular dystrophy; Animal disease; Model; Phenotype

\section{INTRODUCTION}

Progressive muscular dystrophy is a group of diseases characterized by progressive and irreversible degeneration of skeletal muscles (Zatz, 2004). One of these diseases is Duchenne muscular dystrophy (DMD), which is a severe X-linked recessive disorder that shows high morbidity and mortality.

The incidence of DMD is one in 3000 male births (Nichols and Lymn, 1994; PassosBueno and Zatz, 1995), and symptoms appear in the first years of life (3 to 5 years old). At ten years, patients show difficulties in locomotion; at 12 years they become wheelchair-bound, and between 20 and 30 years of age they die (Nichols and Lymn, 1994; Passos-Bueno and Zatz, 1995; Manzur and Muntoni, 2002).

Many treatments have been attempted in the last 20 years, but they all have failed (Hoffman and Dressman, 2001; Dell'Agnola et al., 2004; Liu et al., 2004). New therapies are still under investigation, some of which are based on animal models (Dell'Agnola et al., 2004; McClorey et al., 2006; Kerkis et al., 2008).

Currently, there are two animal models for DMD, namely the $m d x$ mouse model, which is the one most used (Brazeau et al., 1992), and the GRMD (golden retriever muscular dystrophy) canine model (Valentine et al., 1988). The latter is a promising model because besides showing the same clinical signs that have already been characterized in humans (Kornegay et al., 1988; Valentine et al., 1990; Valentine and Cooper, 1991; Nichols and Lymn, 1994; Bergman et al., 2002; Nguyen et al., 2002; Shelton and Engvall, 2002; Collins and Morgan, 2003), these dogs carry the same frameshift mutation that is also seen in some DMD patients (Sharp et al., 1992). Other vertebrate models related to dystrophy have been well described by Vainzof et al. (2008).

\section{MATERIAL AND METHODS}

In 2002, our group established the first and still the only GRMD kennel in Latin America (Ambrosio et al., 2008). Since then, we have been studying and characterizing this group of animals, hoping to better understand the disease in humans and to develop new approaches for treating muscular dystrophy.

Herein, we summarize our findings on important clinical signs and pathological features of 15 GRMD dogs after following these animals very closely from birth. Our observa- 
tions allowed us to identify three different phenotypes, and to classify them as mild, moderate and severe. All 15 GRMD dogs had their affected status confirmed by polymerase chain reaction analysis and genotyping (Figure 1A), according to a protocol described elsewhere (Honeyman et al., 1999; Kerkis et al., 2008).

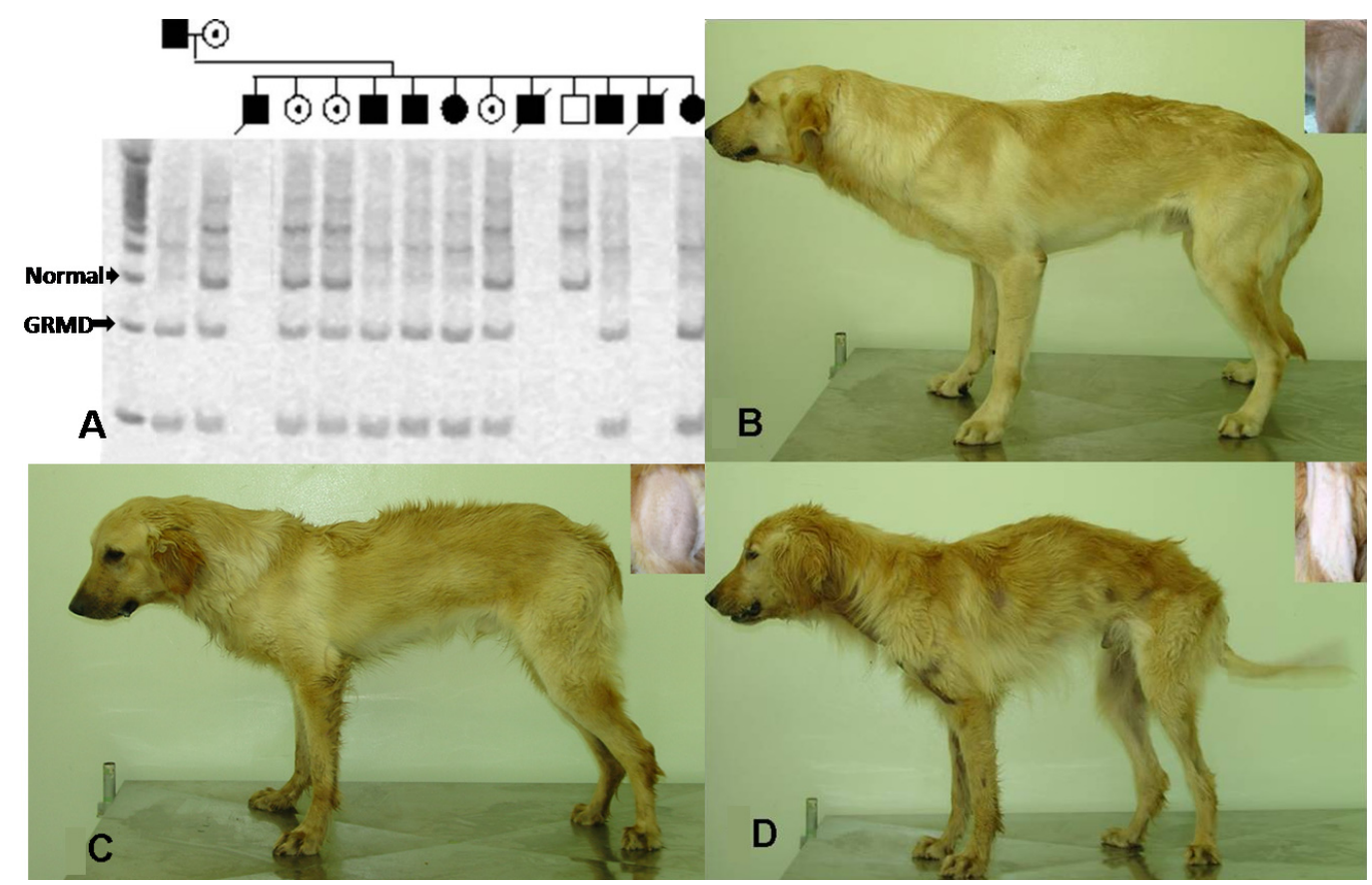

Figure 1. A. Duchenne muscular dystrophy genotyping of golden retriever muscular dystrophy (GRMD) puppies with $\mathrm{X}$-linked muscular dystrophy that were genotyped within $48 \mathrm{~h}$ after birth. The genomic polymerase chain reaction product digested with Sau96I produces the wild-type band $(310 \mathrm{bp})$ and the mutant band (150 bp) labeled with arrows. Squares $=$ affected male. Filled circles $=$ affected female. Open circles $=$ carrier female. B. Grade I phenotype (Suflair dog). C. Grade II phenotype. D. Grade III phenotype showing general hypotrophy. Inset in B-D = thigh muscle mass.

\section{Histological parameters}

Muscle fragments were collected from the vastus lateralis muscle from each of the 15 dogs. To avoid any motor loss of function in the GRMD animals, a single muscle was chosen to represent the pelvic limb. The hind limb is considered to be more affected in the canine dystrophy model (Howell et al., 1997), and the vastus lateralis was described by Childers et al. (2002) as a muscle group that incurs damage and regeneration between 1 and 6 months of age in GRMD dogs.

An open biopsy was performed and a $0.5-\mathrm{cm}^{2}$ fragment was collected from the middle portion of the muscle. After venous cannulation and fluid therapy, the dogs were subjected to an anesthetic protocol (Kerkis et al., 2008). 
Fragments of muscle were cooled with liquid nitrogen and frozen at $-80^{\circ} \mathrm{C}$. Crosssections of 6 to $8 \mu \mathrm{m}$ were made on a Leica Cryostat $\mathrm{CM} 1850$ at $-20^{\circ} \mathrm{C}$ and afterward stained with hematoxylin-eosin.

\section{Creatine kinase analysis}

Blood samples were collected monthly by jugular venipuncture from the GRMD dogs. Serum creatine kinase was determined with an automated analyzer $\left(\right.$ Labtest $^{\mathbb{B}}$ - LABTEST Diagnóstica S.A., Lagoa Santa, MG, Brazil).

\section{RESULTS}

Clinical observations of the entire group of GRMD dogs showed that from birth affected dogs are weaker and more lethargic than either non-affected or carrier dogs. Additionally, affected dogs showed lower daily weight gain and a high risk of developing hypoglycemia, hypothermia and sepsis. Feeding when they are young is difficult because of tongue macroscopic hypertrophy, which results in difficulties in sucking (McKerrell, 2001). Supplementation with a replacement milk product for puppies (Royal Canin first age milk ${ }^{\mathbb{B}}$, Aimargues, France) is done through an orogastric tube. After the initial most critical 15-day postnatal period, animals demonstrated improved sucking and weight gain, showing the first critical period of life.

In general, gastrointestinal alterations are noticed early, which progress in some dogs. In our dogs, dysphagia and excessive salivation were observed when dry food was ingested, which prompted us to replace the dry food with moist food when the animals were seven months old. However, they still presented megaesophagus, which resulted in regurgitation and esophagitis. These dogs also showed a sliding hiatal hernia, which led to emesis as the herniation enlarged. These alterations facilitated the occurrence of aspiration pneumonia in $60 \%$ of the affected dogs in the kennel. Tongue hypertrophy also makes water consumption difficult, which may result in dehydration, and requires the use of fluid therapy in some cases.

The first signs of muscular dystrophy were clearly noticed around the fourth month, when affected dogs showed tarsal overflexion with genu varum and genu valgum (cow's hock) in the hind limbs. At this age, the dogs became thinner and displayed stifle hypomobility while walking. The fore limbs showed lateral rotations of the phalanges, resulting in carpal overflexion with genu varum. Walking or exercise proved to be difficult skills after 6 months of age. As time passed, the limbs became stiff with range of joint motion absent or decreased while moving. Steps became short, with some dogs showing bunny hopping. At around two years of age, dogs can show dilated cardiomyopathy (Pellegrino et al., 2007).

These clinical-pathological findings were observed in most of the GRMD dogs, either by our group or by others (Howell et al., 1997; McKerrell, 2001). However, a close observation of these 15 affected dogs allowed us to classify the phenotypes of these animals into three groups: mild, moderate and severe. The mild phenotype, termed grade I (Figure 1B), included five dogs, which showed less muscular and skeletal symmetry complications, such as less carpal overflexion with genu varum and minimal alterations in hind limbs (inset, Figure 1B), compared to the other dogs. Gastrointestinal alterations were of low grade, such as a discreet esophagus dilatation. Feeding was better than in other GRMD dogs, and these dogs showed no daily episodes of regurgitation (Grando et al., 2009). Cardiac problems and dehydration were 
not observed in this group of dogs. Animals in this group tolerated exercise better than others.

Pathological muscle histology showed typical features of a dystrophic process with variability in fiber size, splitting, degeneration, endomysial fibrosis, fiber regeneration, and necrosis (Figure 2). This degeneration process was observed in all three groups, irrespective of phenotype. Creatine kinase levels were similarly high and variable in all three groups (Figure 2) (Ambrosio et al., 2008; Kerkis et al., 2008).

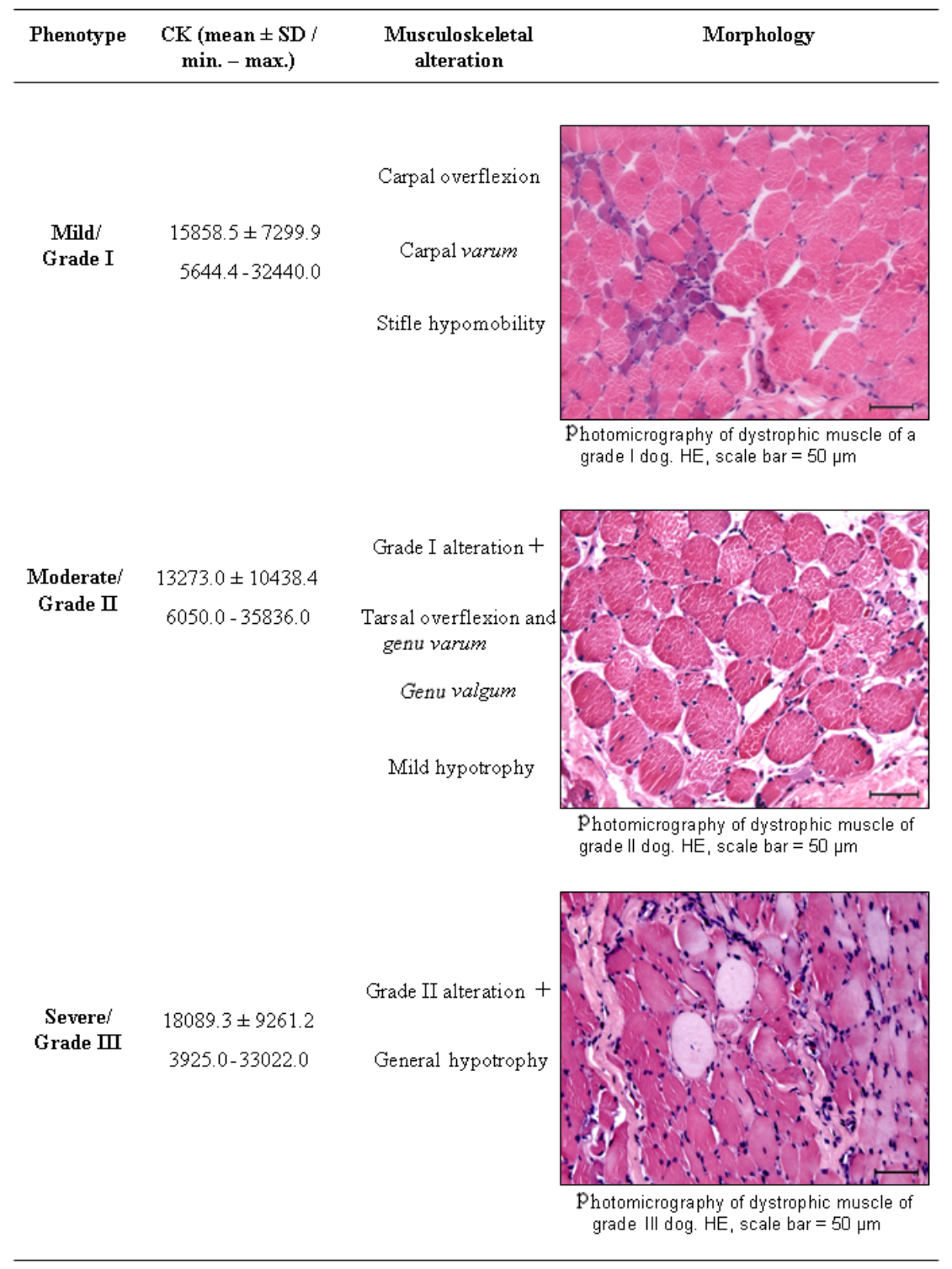

Figure 2. Description of clinical-pathological signs in three different golden retriever muscular dystrophy dog phenotypes. $\mathrm{CK}=$ creatine kinase. 
The moderate phenotype, termed grade II (Figure 1C), included five dogs, which showed more severe clinical signs than those in the grade I group. Animals in this group also showed all the signs described earlier for the grade I group, but with alterations in hind limbs, such as tarsal overflexion with genu varum and genu valgum (cow's hock), which resulted in bunny hopping. In this group, regurgitation was more frequent with dogs displaying two to ten episodes per day, which contributed to dehydration. Sliding hiatal hernia was observed by radiographic examination and showed sliding from 10 to $15 \%$ into thoracic cavity (Alves et al., 2009).

Grade III group showed carpal overflexion and genu varum, stifle hypomobility, tarsal overflexion with genu varum and genu valgum, which is associated with important hypotrophies such as musculoskeletal alterations. The gastrointestinal system showed tongue hypertrophy and megaesophagus. More than ten episodes per day of regurgitation were observed. They showed 30 to $40 \%$ sliding hiatal hernia, which was characterized by cardia, fundus and body herniation into the thoracic cavity (Figure 1D).

\section{DISCUSSION}

The identification and classification of these three phenotypes may reflect important inter- and intra-familiar clinical variability, which is seen in DMD patients and which cannot be attributed to genetic factors (Vainzof and Zatz, 2003), but a correlation between the amount of dystrophin and the severity of the phenotype has been suggested by the cited authors. Sifringer et al. (2004) suggested that an increase in some proteins such as casein kinase I, dynactin 3 light chain and the core-binding factor beta, which are involved in the regulation of the cell cycle, may be related to a milder phenotype. Future studies should be carried out to establish if a difference in the levels of dystrophin can also be observed in these dogs and whether these levels may explain the different phenotypes described here.

DMD has a milder variant known as Becker muscular dystrophy (BMD). BMD is less severe but displays variable phenotypes, ranging from a slightly less severe DMDlike condition to a very mild condition. Some patients may be able to walk throughout their lives. A patient is classified as having DMD when wheelchair-dependent before the age of 13, and as having BMD when able to walk beyond the age of 16 (Vainzof and Zatz, 2003). However, a recent clinical casebook described a boy without any signs of DMD (Dubowitz, 2006) but with confirmation of molecular findings. Both forms of muscular dystrophy are found in patients carrying similar mutations, which indicates that the phenotype cannot be determined solely by the genomic mutation in the Xp21 locus; other factors may also play a role.

A similar situation is observed in GRMD dogs (Ambrosio et al., 2008). We propose the hypothesis that the milder phenotype described here is due to a modifier gene most likely inherited through male-to-male transmission. It is important to note that in our kennel we also have a number of affected female dogs that carry the same mutation in both X chromosomes; however, no variation in phenotype is seen among affected females. Therefore, it seems that the variation we observed among these affected dogs is somehow limited to males. Understanding the mechanism and genetic bases underlying these variations is of great interest (Ambrosio et al., 2007). 


\section{CONCLUSIONS}

In summary, we identified three phenotypes of the GRMD dog, which is a promising animal model for DMD because these dogs show most of the signs found in the human disease, except the respiratory symptoms and loss of gait. However, the use of GRMD dogs in pre-clinical trials must be carried out carefully as the existence of different phenotypes may account for different responses to drugs and treatments. Therefore, the clinical outcome of therapeutic trials with GRMD dogs should be interpreted with caution.

\section{Conflict of interest statement}

None of the authors of this paper has a financial or personal relationship with other people or organizations that could inappropriately influence or bias the content of the study.

\section{REFERENCES}

Alves FR, Feitosa MLT, Gatti A, Fadel L, et al. (2009). Radiologic images of the thoracic cavity of Golden Retriever dogs affected by muscular dystrophy. Pesq. Vet. Bras. 29: 99-104.

Ambrosio CE, Zucconi E, Martins D, Vanucchi C, et al. (2007). Extreme clinical variability in GRMD: From neonatal death to asymptomatic carriers. Neuromuscul. Disord. 17: 776.

Ambrosio CE, Valadares MC, Zucconi E, Cabral R, et al. (2008). Ringo, a Golden Retriever Muscular Dystrophy (GRMD) dog with absent dystrophin but normal strength. Neuromuscul. Disord. 18: 892-893.

Bergman RL, Inzana KD, Monroe WE, Shell LG, et al. (2002). Dystrophin-deficient muscular dystrophy in a Labrador retriever. J. Am. Anim. Hosp. Assoc. 38: 255-261.

Brazeau GA, Mathew M and Entrikin RK (1992). Serum and organ indices of the $m d x$ dystrophic mouse. Res. Commun. Chem. Pathol. Pharmacol. 77: 179-189.

Childers MK, Okamura CS, Bogan DJ, Bogan JR, et al. (2002). Eccentric contraction injury in dystrophic canine muscle. Arch. Phys. Med. Rehabil. 83: 1572-1578.

Collins CA and Morgan JE (2003). Duchenne's muscular dystrophy: animal models used to investigate pathogenesis and develop therapeutic strategies. Int. J. Exp. Pathol. 84: 165-172.

Dell'Agnola C, Wang Z, Storb R, Tapscott SJ, et al. (2004). Hematopoietic stem cell transplantation does not restore dystrophin expression in Duchenne muscular dystrophy dogs. Blood 104: 4311-4318.

Dubowitz V (2006). Enigmatic conflict of clinical and molecular diagnosis in Duchenne/Becker muscular dystrophy. Neuromuscul. Disord. 16: 865-866.

Grando AP, Mariana ANB, Miglino MA, Sterman FA, et al. (2009). Abdominal and pelvic ultrasonography in healthy golden retriever dogs, carriers and affected by gradual muscular dystrophy. Cienc. Rural 39: 123-128.

Hoffman EP and Dressman D (2001). Molecular pathophysiology and targeted therapeutics for muscular dystrophy. Trends Pharmacol. Sci. 22: 465-470.

Honeyman K, Carville KS, Howell JM, Fletcher S, et al. (1999). Development of a snapback method of single-strand conformation polymorphism analysis for genotyping Golden Retrievers for the X-linked muscular dystrophy allele. Am. J. Vet. Res. 60: 734-737.

Howell JM, Fletcher S, Kakulas BA, O'Hara M, et al. (1997). Use of the dog model for Duchenne muscular dystrophy in gene therapy trials. Neuromuscul. Disord. 7: 325-328.

Kerkis I, Ambrosio CE, Kerkis A, Martins DS, et al. (2008). Early transplantation of human immature dental pulp stem cells from baby teeth to golden retriever muscular dystrophy (GRMD) dogs: Local or systemic? J. Transl. Med. 6: 35.

Kornegay JN, Tuler SM, Miller DM and Levesque DC (1988). Muscular dystrophy in a litter of golden retriever dogs. Muscle Nerve 11: 1056-1064.

Liu J, Okamura C, Bogan D, Bogan J, et al. (2004). Effects of prednisone in canine muscular dystrophy. Muscle Nerve 30: 767-773.

Manzur AY and Muntoni F (2002). The management of Duchenne muscular dystrophy. Curr. Paediatr. 12: 261-268.

McClorey G, Moulton HM, Iversen PL, Fletcher S, et al. (2006). Antisense oligonucleotide-induced exon skipping restores 
dystrophin expression in vitro in a canine model of DMD. Gene Ther. 13: 1373-1381.

McKerrell RE (2001). Miopatias Caninas e Felinas. In: Tratado de Medicina de Pequenos Animais (Dunn JK, ed.). Roca, São Paulo, 699-702.

Nguyen F, Cherel Y, Guigand L, Goubault-Leroux I, et al. (2002). Muscle lesions associated with dystrophin deficiency in neonatal golden retriever puppies. J. Comp Pathol. 126: 100-108.

Nichols PL and Lymn RW (1994). Gene Therapy in Duchenne Muscular Dystrophy. NIH Guide. Available at [http:// grants2.nih.gov/grants/guide/pa-files/PA-94-040.html]. Accessed April 2008.

Passos-Bueno MR and Zatz M (1995). Miopatias Hereditárias: Avanços dos Últimos dois anos. In: Condutas em Neurologia (Nitrini R, Machado LR, Targas EM and Rabello GD, eds.). Clínica Neurológica HC/FMUSP, São Paulo, 70-78.

Pellegrino A, Petrus LC, Pereira GG, Soares EC, et al. (2007). Standardization of echocardiographic parameters of healthy Golden Retriever dogs. Cienc. Rural 37: 1039-1044.

Sharp NJ, Kornegay JN, Van Camp SD, Herbstreith MH, et al. (1992). An error in dystrophin mRNA processing in golden retriever muscular dystrophy, an animal homologue of Duchenne muscular dystrophy. Genomics 13: 115-121.

Shelton GD and Engvall E (2002). Muscular dystrophies and other inherited myopathies. Vet. Clin. North Am. Small Anim. Pract. 32: 103-124.

Sifringer M, Uhlenberg B, Lammel S, Hanke R, et al. (2004). Identification of transcripts from a subtraction library which might be responsible for the mild phenotype in an intrafamilially variable course of Duchenne muscular dystrophy. Hum. Genet. 114: 149-156.

Vainzof M and Zatz M (2003). Protein defects in neuromuscular diseases. Braz. J. Med. Biol. Res. 36: 543-555.

Vainzof M, Ayub-Guerrieri D, Onofre PC, Martins PC, et al. (2008). Animal models for genetic neuromuscular diseases. J. Mol. Neurosci. 34: 241-248.

Valentine BA and Cooper BJ (1991). Canine X-linked muscular dystrophy: selective involvement of muscles in neonatal dogs. Neuromuscul. Disord. 1: 31-38.

Valentine BA, Cooper BJ, de Lahunta A, O'Quinn R, et al. (1988). Canine X-linked muscular dystrophy. An animal model of Duchenne muscular dystrophy: clinical studies. J. Neurol. Sci. 88: 69-81.

Valentine BA, Cooper BJ, Cummings JF and de Lahunta A (1990). Canine X-linked muscular dystrophy: morphologic lesions. J. Neurol. Sci. 97: 1-23.

Zatz M (2004). Genômica das Doenças Neuromusculares e Neurodegenerativas. In: Genômica (Mir L, ed.). Atheneu, São Paulo, 345-360. 